ANL/CHM/CP-99602

\title{
MOLECULAR DYNAMICS SIMULATION OF IMPURITIES IN NANOCRYSTALLINE DIAMOND GRAIN BOUNDARIES*
}

Michael Sternberg ${ }^{1}$, Peter Zapol ${ }^{2}$, Thomas Frauenheim ${ }^{1}$, Dieter M. Gruen ${ }^{2}$, and Larry A. Curtiss ${ }^{2}$

${ }^{1}$ Universität-GH Paderborn, Fachbereich Physik, Theoretiche Physik, d-33098, Paderborn, Germany

${ }_{2}^{2}$ Materials Science and Chemistry Divisions, Argonne National Laboratory, Argonne, IL 60439

submitted for publication in the

Proceedings of Symposium U: Amorphous and Nanostructured Carbon

Materials Research Society Fall 1999 Meeting

Boston, Massachusetts

November 29-December 3, 1999

The submitted manuscript has been created by
the University of Chicago as Operator of
Argonne National Laboratory ("Argonne") under
Contract No. W-31-109-ENG-38 with the U.S.
Department of Energy. The U.S. Government
retains for itself, and others acting on its behalf,
a paid-up, nonexclusive, irrevocable worldwide
license in said article to reproduce, prepare
derivative works, distribute copies to the public,
and perform publicly and display publicly, by
or on behalf of the Government.

*Work supported by the U.S. Department of Energy, BES-Materials Sciences, under Contract W-31-109-ENG-38. 


\title{
MOLECULAR DYNAMICS SIMULATION OF IMPURITIES IN NANOCRYSTALLINE DIAMOND GRAIN BOUNDARIES.
}

\author{
Michael Sternberg ${ }^{\S}$, Peter Zapol ${ }^{\ddagger}$, Thomas Frauenheim ${ }^{\S}$, Dieter M. Gruen ${ }^{\ddagger}$, and Larry A. Curtiss ${ }^{\ddagger}$ \\ ${ }^{\ddagger}$ Materials Science and Chemistry Divisions, Argonne National Laboratory, Argonne, IL 60439 \\ ${ }^{\S}$ Universität-GH Paderborn, Fachbereich Physik, Theoretiche Physik, D-33098 Paderborn, \\ Germany
}

\section{ABSTRACT}

Nanocrystalline diamond films grown on $\mathrm{Si}$ substrates at $800^{\circ} \mathrm{C}$ from hydrogen-poor plasmas have a number of highly desirable mechanical and electronic properties. Impurities were found by SIMS measurements to be uniformly distributed throughout the thickness of the films at a level of $10^{17}-10^{18} \mathrm{~cm}^{-3}$. It is likely that the impurities are located at the grain boundaries, which play a crucial role in controlling important characteristics of the films, such as electrical conductivity and electron emission. Density-functional based tight-binding (DFTB) molecular dynamics simulations were performed for diamond high-energy high-angle (100) twist grain boundaries with impurities such as $\mathrm{N}, \mathrm{Si}$ and $\mathrm{H}$.

\section{INTRODUCTION}

In contrast to diamond films grown by the traditional method involving $\mathrm{CH}_{4} / \mathrm{H}_{2}$ mixtures, where micron-size crystallites typically constitute the microstructure of the films, diamond grown from argon plasmas containing carbon is characterized by a microstructure consisting of crystallites with an average size of 3-10 nm. ${ }^{1}$ This nanocrystalline diamond can be grown to thicknesses of at least $30 \mu \mathrm{m}$ from argon/fullerene or argon/methane microwave plasmas. A change in electrical conductivity is observed with reduction in crystallite size, with the material going from an insulator to an electrical conductor. This phenomenon is largely due to the fact that grain boundary carbon atoms are $\pi$-bonded. ${ }^{2}$ The grain boundaries are therefore conducting, and because their numbers vastly increase with decreasing crystallite size, the entire film becomes electrically conducting. Impurities are introduced into the films during the growth process and are evenly distributed throughout the films. It is plausible that impurities are predominantly concentrated in the grain boundaries and take part in electrical conduction. It was shown experimentally that films grown in plasmas with high nitrogen concentration have better electron emission properties and higher conductivity.

The impurity-free grain boundaries in nanocrystalline diamond have been the subject of several theoretical studies. The structure of twist grain boundaries in diamond was studied by Keblinski $e t a l^{2}$ using Tersoff interatomic potentials. They found that (100) grain boundaries are more stable against decohesion than (110) and (111) grain boundaries. Up to $80 \%$ of the $\Sigma 29$ grain boundary carbon atoms were found to be three-coordinated. A tight-binding molecular dynamics study of the $\Sigma 29$ grain boundary by Cleri et al. ${ }^{3}$ indicated that about $40 \%$ of the grain boundary atoms are three-coordinated and that the electronic band structure has a broad spectrum of gap states between which a hopping conduction is possible. We recently published results ${ }^{4}$ of a periodic density functional calculation of a stacking fault that is a model for the diamond grain boundary with $\mathrm{sp}^{2}$ bonding across the interface. The electronic structure of diamond with defects is characterized by the presence of $\pi$-states in the forbidden gap that are localized on the interfacial atoms. These states produce several broad peaks in the gap. 
In this paper we report on new density-functional based tight-binding molecular dynamics calculations of the structure of a $\Sigma 13$ twist (100) grain boundaries with N, H and Si impurities in diamond.

\section{THEORETICAL METHODS}

The molecular dynamics calculations were performed using a density functional based tight binding (DFTB) method with self-consistent charges using the approach of Frauenheim, Seifert, et al. ${ }^{5,6}$ This DFTB method uses a linear combination of atomic orbitals (LCAO) formalism and a local density approach. In this scheme the Kohn-Sham orbitals of the many-atom problem are expressed in terms of an explicit minimal basis set of atom-centered localized valence electron orbitals. The general eigenvalue problem for the many-atom configuration is solved to determine the single particle energies and eigenstates of the system, neglecting contributions of the threecenter and crystal-field integrals to the matrix elements. The total energy of a system is written as a sum over a "band structure energy" and a short-range repulsive two-particle interaction term. The latter term is derived from a universal pair-potential resulting from a fitting of potential energy curves from self-consistent field calculations in the local density approximation. These parameters have been determined for carbon previously. ${ }^{5}$ Interatomic forces for molecular dynamics can be calculated from analytical expressions for the gradients of the total energy at the atomic positions. The self-consistent charge scheme is based on the second order term in an expansion of energy over electron density fluctuations. This term is expressed through Mulliken charges and calculated self-consistently at each simulation step.

\section{RESULTS}

The DFTB molecular dynamics calculations were done on the $\Sigma 13$ twist (100) grain boundaries in diamond. A relative rotation of the two halves of the crystal by $67.4^{\circ}$ about the zaxis gives a planar cell with 13 atoms per (001) plane. A periodic model of the grain boundary was constructed using two grain boundaries per unit cell. Each repeating cell has a thickness of 16 layers and thus contains 208 carbon atoms. Molecular dynamics simulation of the initial structure was performed at $1500 \mathrm{~K}$ (approximately corresponding to experimental growth temperatures) for $0.5 \mathrm{ps}$. Subsequently, simulated annealing was performed at gradually lower temperatures and the final structure was optimized by a conjugate gradient method. Structural disorder is confined to the two grain boundary planes and the rest of the diamond crystal remains nearly perfectly ordered. Threefold-coordinated atoms constitute $46 \%$ of the two grain boundary planes, similar to the tight-binding molecular dynamics results reported by Cleri et al.$^{3}$ About half of the three-coordinated atoms have an $\mathrm{sp}^{2}$ configuration, i.e. have bond angles close to $120^{\circ}$ with their neighbors, and the other half have a tetrahedral arrangement of their three bonds, leading to a geometry configuration that is typical for a carbon atom with a dangling bond.

For the study of impurities, a carbon atom in the interface layer of the grain boundary was substituted by nitrogen in the annealed structure and the annealing procedure was fully repeated to find the optimized position of the nitrogen atom. The most stable nitrogen position was obtained starting from a three-coordinated site with nonplanar arrangement of the bonds (see, Fig. 1). It is an expected result considering that such arrangement is favored for substitutional nitrogen in diamond bulk. Other initial positions, including a planar three-coordinated site (true $\mathrm{sp}^{2}$ carbon) and a four-coordinated site ( $\mathrm{sp}^{3}$ carbon), lead to a quite similar nonplanar threecoordinated nitrogen configuration with bond angles from $99^{\circ}$ to $148^{\circ}$. Generally, the carbonnitrogen bond lengths are in the range $1.45 \AA-1.56 \AA$, and occasionally one $\mathrm{C}$ - $\mathrm{N}$ bond is (up to 


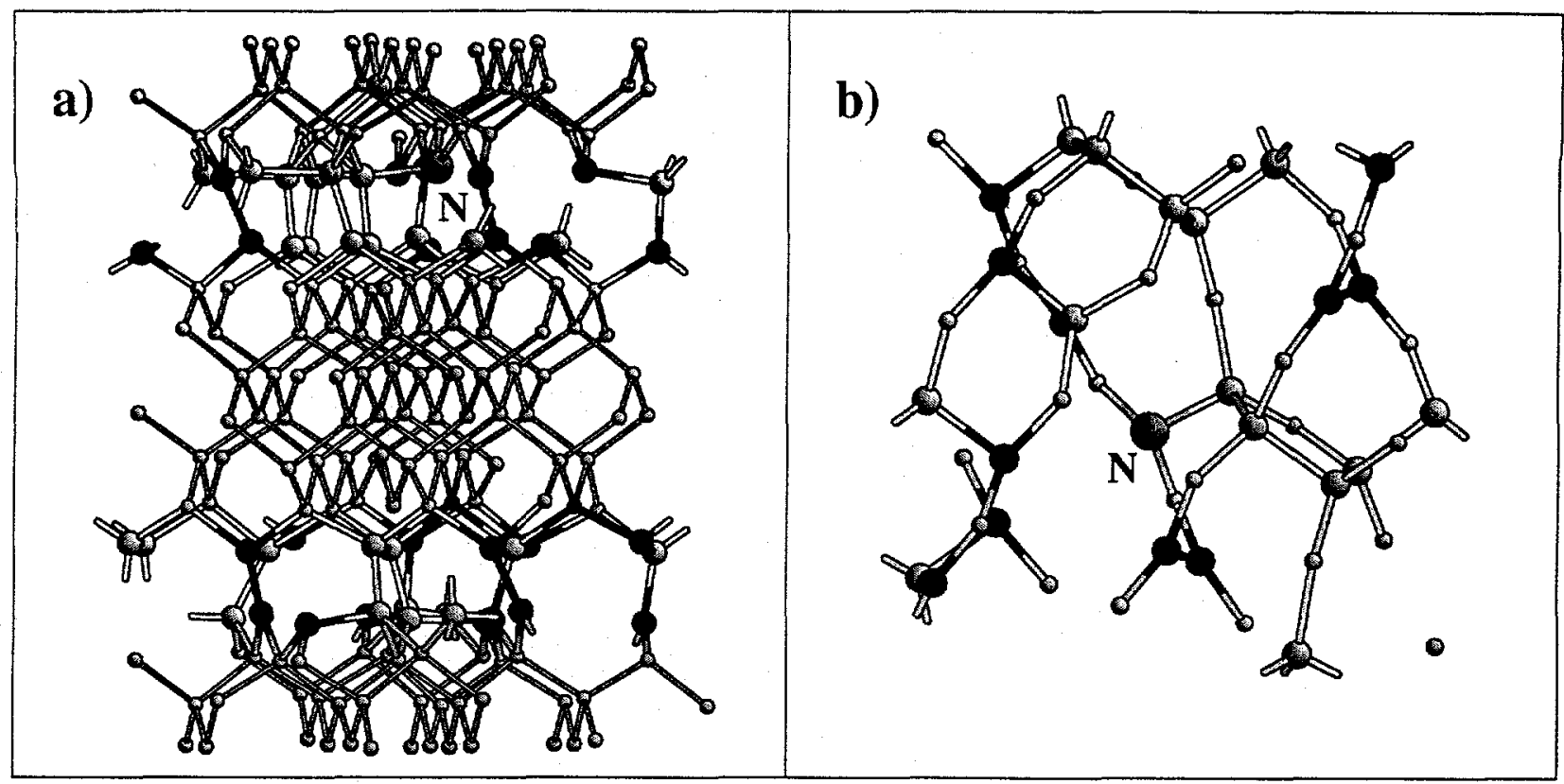

Figure 1. Side view(a) and top view of the four interface layers(b) of the periodic cell for an optimized $\Sigma 13$ grain boundary with nitrogen impurity. Two grain boundaries are shown in (a). Black atoms are three-coordinated and gray atoms are four-coordinated. Atoms in the first layers of the interfaces are shown as larger spheres. Bonds extending across the cell boundary are shown as half bonds.

$2.0 \AA$ ) than the other two bonds. The distortions of bonds are caused by the restrictions on geometry due to the surrounding carbon lattice. These restrictions are weaker for nitrogen in the grain boundary compared to the diamond bulk because of the local disorder. Therefore, it is easier to accommodate nitrogen in the grain boundary. Indeed, the calculated formation energy of substitutional defect in the grain boundary for the most stable configuration is $0.5 \mathrm{eV}$ compared to $6.1 \mathrm{eV}$ in the diamond single crystal. These formation energies were calculated as the work to bring a nitrogen atom from infinity to the system minus the work to remove a carbon atom from the system to infinity. Formation energies for less favorable nitrogen substitutions were up to $3 \mathrm{eV}$ higher.

The optimized structure of a grain boundary with a substituted silicon atom is shown in Fig. 2. Silicon substitution into grain boundary sites always results in a four-coordinated $\mathrm{Si}$ atom independent of the initial configuration. Carbon - silicon bond lengths are typically $1.78 \AA$ to $2.01 \AA$. Bond angles are distorted from tetrahedral, with values between $84^{\circ}$ and $110^{\circ}$. The formation energies of the substitutional $\mathrm{Si}$ are $2.9 \mathrm{eV}$ to $5.2 \mathrm{eV}$. This is less favorable compared to nitrogen substitution energies. The energy required to insert $\mathrm{Si}$ into the grain boundary is still much lower than $8.2 \mathrm{eV}$ required to insert it into the diamond crystal. A grain boundary has much lower local density than the ordered diamond structure and, therefore, it is easier to accommodate a larger silicon atom. The number of four-coordinated carbon atoms in the vicinity of Si increases. In the timescale of simulation (about $1 \mathrm{ps}$ ) neither silicon nor nitrogen atoms move between different sites. 
a)

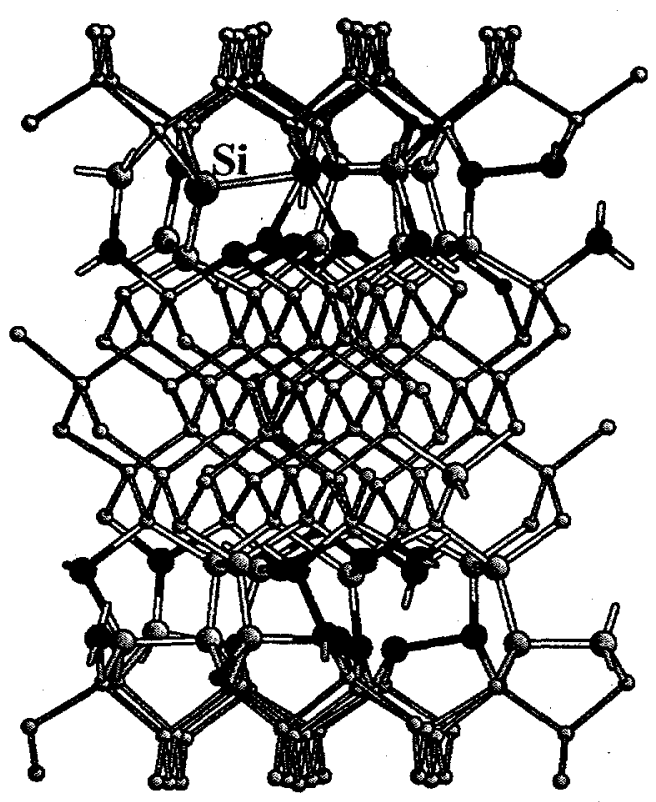

b)



Figure 2. Side view(a) and top view of the four interface layers(b) of the periodic cell for an optimized $\Sigma 13$ grain boundary with silicon impurity. Atom appearance is the same as in Fig.1.

The last impurity studied, hydrogen, was simulated by adding four hydrogen atoms per grain boundary unit cell at random positions in the interface of annealed structure. As in previous simulations, the annealing procedure was repeated. All hydrogens are bonded to carbon atoms in the final structure. All of these carbon atoms are four-coordinated. The average carbon hydrogen bond length is about $1.10 \AA$. The average hydrogen binding energy is $3.5 \mathrm{eV}$. Thus, hydrogens saturate dangling bonds of the three-coordinated carbons, as expected. At the beginning of the simulation $(1500 \mathrm{~K}-1200 \mathrm{~K})$ hydrogens move among different carbon sites in the grain boundary plane whereas at lower temperatures $(500 \mathrm{~K}$ and lower) their motion is restricted to a vicinity of one carbon atom.

\section{CONCLUSIONS}

In this paper we report on calculations of silicon, nitrogen and hydrogen impurities in the $\Sigma 13$ twist (100) grain boundaries in diamond. Nitrogen substitution requires $0.5-3.5 \mathrm{eV}$ and results in a three-coordinated nonplanar nitrogen configuration. Silicon substitution is less favorable $(2.9 \mathrm{eV}-5.2 \mathrm{eV})$ and always results in a four-coordinated Si atom. Hydrogen saturates the dangling bonds of carbon atoms in the grain boundary. It is found that insertion of any of these impurities into the grain boundary is always easier than into the bulk diamond.

\section{ACKNOWLEDGMENT}

We gratefully acknowledge use of the advanced computing resources at Argonne's Center for Computational Science and Technology. M. S. acknowledges support from Deutsche Forschungsgemeinschaft. This work is supported by the U.S. Department of Energy, BESMaterials Sciences, under Contract W-31-109-ENG-38. 


\section{REFERENCES}

1. C. Zuiker, A. K. Krauss, D. M. Gruen, X. Pan, J. C. Li, R. Csencsits, A. Erdemir, C. Bindal, and G. Fenske, Thin Solid Films, 270, 154 (1995):

2. P. Keblinski, D. Wolf, S. R. Phillpot, and H. Gleiter, J. Mater. Res., 13, 2077 (1998).

3. F. Cleri, P. Keblinski, L. Colombo, D. Wolf, S. R. Phillpot, Europhys. Lett., 46, 671 (1999).

4. D. M. Gruen, P. C. Redfern, D. A. Horner, P. Zapol, and L. A. Curtiss, J. Phys. Chem., 103, 5459 (1999).

5. D. Porezag, Th. Frauenheim, Th. Kohler, G. Seifert, and R. Kaschner, Phys. Rev. B, 51, 12947 (1995).

6. M. Elstner, D. Porezag, G. Jungnickel, J. Elsner, M. Haugk, Th. Frauenheim, S. Suhai, G. Seifert, Phys. Rev. B, 58, 7260 (1998). 